\title{
Objective evaluation of clinical outcomes of laparoscopy-assisted pylorus-preserving gastrectomy for middle-third early gastric cancer
}

\author{
Xiang $\mathrm{Xia}^{\dagger}{ }^{\dagger}$ Jia Xu ${ }^{\dagger}$, Chunchao Zhu, Hui Cao, Fengrong Yu ${ }^{*}$ and Gang Zhao ${ }^{*}$ (1)
}

\begin{abstract}
Background: Laparoscopic-assisted pylorus-preserving gastrectomy (LAPPG) is a minimally invasive functionpreserving surgery for early gastric cancer. This study was designed to investigate the clinical outcomes between LAPPG and laparoscopy-assisted distal gastrectomy (LADG) by objective evaluation.

Methods: A total 167 pT1NOMO gastric cancer patients underwent LAPPG $(n=70)$ and LADG $(n=97)$ were retrospectively analyzed. By evaluating the functional advantages, objective short-term and one year follow-up outcomes were compared.

Results: There is no significant difference in perioperative clinical characteristics as well as pathologic results between LAPPG and LADG group while the cost is higher in latter $(p=0.004)$. The Clavien-Dindo grade II or higher complications were 15.7 and $13.4 \%$ in LAPPG and LADG group respectively $(p=0.824)$. In one year follow-up, nutritional status was significantly better in LAPPG group accompanied by better pylorus function preserving.
\end{abstract}

Conclusion: LAPPG is an acceptable surgical procedure for pT1NOMO middle portion gastric cancer patients in terms of nutritional and economic advantage.

Trial registration: Chinese Clinical Trial Registry (ChiCTR-PIC-17012358, Date of Registration:2017-08-14).

Keywords: Laparoscopic-assisted pylorus-preserving gastrectomy, pT1NOM0 gastric cancer, Gallstone, Gastric emptying, Nutritional status

\section{Background}

Gastric cancer is the third most frequent cause of cancer related death and the fifth most common cancer worldwide with nearly 951,000 newly diagnosed patients as well as 723,000 death in 2012 [1]. In recent years, due to the popularization of heath screening programs and development of high-quality endoscopic instruments, the number of early gastric cancer (EGC) has been gradually increasing. Furthermore, because EGC usually owns a low metastatic incidence and favorable survival rates, surgeons have started to place special emphasis on

\footnotetext{
*Correspondence: yufengrongrj@163.com; zhaogangrj@163.com

${ }^{+}$Xiang Xia and Jia Xu contributed equally to this work.

Department of Gastrointestinal Surgery, Ren Ji Hospital, School of Medicine, Shanghai Jiao Tong University, No. 1630, Dongfang Road, Shanghai 200127 People's Republic of China
}

function-preserving and nutritional status improvement for those patients $[2,3]$.

Compared to traditional open gastrectomy, laparoscopic gastrectomy has the superiority of the minimally invasive approach, such as less postoperative pain, better cosmetic results, early recovery of bowel function and a rapid recovery to regular activity [4-6]. Moreover, laparoscopy-assisted distal gastrectomy (LADG) has been a standard surgical procedure for Stage I gastric cancer [7]. Nevertheless, because the extent of distal gastrectomy is identical in laparoscopic and open surgery, the long-term outcomes and postgastrectomy symptoms of LADG, including dumping syndromes or remnant gastritis were similar to open distal gastrectomy.

Pylorus-preserving gastrectomy (PPG) is a typical operation of function-preserving for EGC located in the 
middle portion of the stomach $[3,8]$. This surgery was first introduced to treat benign gastric ulcers in 1967 by Maki et al. [9]. And in the current version of the Japanese Gastric Cancer Treatment Guidelines, PPG is described as a modified procedure for cT1N0M0 EGC in the middle portion of the stomach [10]. Based on the advantages of laparoscopy and PPG, LAPPG started to apply in some EGC patients by some gastrointestinal surgeons. But to date, no randomized controlled trial was reported to compare the perioperative outcomes and long-term nutritional status between LAPPG and LADG.

In this study, we present the short-term outcomes and one-year follow up postoperative nutritional status including gallstone formation and gastric emptying evaluation of LAPPG and LADG.

\section{Methods}

\section{Patients}

Between April 2015 and December 2017, A review of medical records including clinical and pathologic reports at Department of Gastrointestinal Surgery, Ren Ji hospital, School of Medicine, Shanghai Jiao Tong University identified 167 pT1N0M0 patients underwent LAPPG and LADG. The indication for LAPPG was cT1N0M0 gastric cancer located in the middle or lower-third of the stomach without evidence of regional lymph node metastasis, more than $5 \mathrm{~cm}$ proximal to the pyloric ring and with a maximum diameter less than $5 \mathrm{~cm}$, while other cT1N0M0 gastric cancer located in the middle or lower-third of the stomach without evidence of regional lymph node metastasis were included in LADG group. All patients received an upper gastrointestinal endoscopy, pathological biopsy, computed tomography, and sometimes endoscopic or abdominal ultrasonography. cT1N0M0 gastric cancer patients would be excluded if they were found later to be $\mathrm{pN} 1$. Whether a patient was treated by LAPPG or LADG was decided by patient request when tumor located more than $5 \mathrm{~cm}$ proximal to the pyloric ring and with a maximum diameter less than $5 \mathrm{~cm}$, and otherwise by attending surgeon preference. Patients who were candidates for endoscopic resection were not included in the study. No patients were received chemotherapy in this study. It was likewise registered in the Chinese Clinical Trial Register (ChiCTR), a primary register of the WHO International Clinical Trials Registry Platform (SN. ChiCTR-PIC-17012358).

\section{Surgical procedures}

After general anesthesia, patients were laid in the supine, reverse Trendelenburg position with leg elevation. A 12-mm trocar for the camera port was inserted through the umbilical port and a pneumoperitoneum was created by $\mathrm{CO}_{2}$ inflation at the pressure of $12 \mathrm{mmHg}$. Under the view of the laparoscopic image, a $12 \mathrm{~mm}$ trocar and a 5 $\mathrm{mm}$ trocar were inserted in the left upper and lower quadrants while two $5 \mathrm{~mm}$ trocar were inserted in the right upper and lower quadrants respectively. Then falciform ligation was lifted by prolene stay sutures.

\section{LAPPG and LADG}

LAPPG partially preserved the greater omentum and cut the omentum $3-4 \mathrm{~cm}$ inferior to the gastroepiploic arcade. Kocher's maneuver to mobilize the duodenum was done to minimize the tension for the gastro-gastric anastomosis. Lymph node included No. 4d(right astroepiploic artery) and No. 4sb(left gastroepiploic artery) were dissected in this procedure. Then the origin of the right gastroepiploic artery and vein were divided carefully and the ligation of the right epiploic vessels should be distal to the branches of infrapyloric vessels to maintain the blood supply to pyloric cuff followed by No. 6 lymph nodes dissection. The No. 5 lymph nodes were left intact with the right gastric vessels was ligated approximately $3 \mathrm{~cm}$ apart from the pylorus. Subsequently No. 7, 8a, 9 and partial 11p lymph nodes were dissected as well as ligation of the left gastric artery. The No. 1, 3 lymph nodes were removed followed by preservation of the hepatic branch of the vagus. After lymphadenectomy, the stomach was extracted through a $5 \mathrm{~cm}$ midline incision. The distal part of the stomach was resected leaving at least $3 \mathrm{~cm}$ antral cuff. The proximal portion of the stomach was kept with a $3 \mathrm{~cm}$ proximal margin for an oncologically safe margin. A two-layer extracorporeal handsewn gastrogastroanastomosis was then performed.

LADG procedure has been described previously [11]. In brief, trocar placement and laparoscopic procedures were similar to LAPPG, except for the dissection of suprapyloric and infrapyloric lymph nodes and preserving the hepatic branch of the vagus nerve. Suprapyloric lymph nodes were completely dissected with the division of the root of the right gastric vessels. Infrapyloric lymph nodes were completely dissected with the division of the root of the right gastroepiploic and infrapyloric vessels. The celiac branch of the vagus nerve was dissected during the dissection of the lymph nodes along the celiac artery. The posterior wall of the remnant stomach and the duodenal stump was mechanically anastomosed with Bilroth I anastomosis.

\section{Postoperative data and surveillance}

Demographic and clinicopathologic data were reviewed from our medical records. The microscopic classification of tumors was based on the 3rd English edition of the Japanese Classification of Gastric Carcinoma [12] and the 7th edition of the International Union Against Cancer/American Joint Committee on Cancer TNM staging 
system [13]. Postoperative complications were categorized according to the Clavien-Dindo classification [14].

In follow up protocol, all patients were examined by physical and blood cell counts, blood chemistry tests including hemoglobin, serum total protein and serum albumin every 6 months. Meanwhile, BMI calculation, computed tomography, abdominal ultrasonography for gallstone, gastric emptying test, tumor markers and gastroscopy were performed at every 6 months hospital visits.

\section{Gallbladder and gastric emptying examination}

Gallbladder volumes were calculated using the ellipsoid method using the formula $\mathrm{V}=0.52(\mathrm{~L} \times \mathrm{W} \times \mathrm{H})$, where $\mathrm{W}$ is the gallbladder width, $\mathrm{H}$ is height and $\mathrm{L}$ is axial length [15]. After an overnight fasting, the gallbladder basal volume was measured in the morning with patients in the supine position turned partially on their right side. The residual volume was measured one hour later after eating two fried eggs. The gallbladder emptying rate was considered the difference between basal volume and residual volume/ basal volumex 100 .

After $12 \mathrm{~h}$ fasting, patients underwent gastric emptying scintigraphy technique by eating a meal of $40 \mathrm{~g}$ of black sesame paste and a $17.5 \mathrm{~g}$ cheese mixed with $300 \mathrm{ml}$ water. The meal was labeled with ${ }^{99 \mathrm{~m}} \mathrm{Tc}$-DTPA $2 \mathrm{mCi}$. Gastric emptying was followed by continuous imaging at $0,5,10,15,20,25,30,60$ and 90 min per frame. We set a linear rate of emptying to the data from 0 to $90 \mathrm{~min}$ and obtained an extrapolated half-time of emptying (normal $\mathrm{t}^{1} \frac{1}{2}=65-85 \mathrm{~min}$ ). Normal values for this meal and imaging methodology were obtained from 20 normal volunteers (age range: 26-60) in 2014 at our hospital (not published data).

Because different feeding times and food components would affect these two examinations, the gastric and gall bladder emptying tests were performed in two separate visits.

\section{Statistical analysis}

SPSS version 13.0 software (SPSS, Chicago, IL, USA) for windows was utilized to perform statistical analyses. Baseline characteristics were compared by Mann-Whitney U-test for continuous variables, and the chi-square test was used for categorical variables. $P$ values less than 0.05 were considered significant.

\section{Results}

\section{Patients' clinicalpathological characteristics and perioperative outcomes}

Detailed information concerning those 167 patients was presented in Tables 1 and 2. The mean age, gender, mean BMI, preoperative comorbidity, ASA (American Society of Anesthesiologists) and ESD history were
Table 1 Characteristics of patients undergoing LAPPG and LADG

\begin{tabular}{llll}
\hline Variables & LAPPG $(n=70)$ & LADG $(n=97)$ & $P$ value \\
\hline Mean age (years) & & & \\
Gender & $56.8 \pm 10.9$ & $57.5 \pm 12.1$ & 0.667 \\
$\quad$ Male & $46(65.7 \%)$ & $63(64.9 \%)$ & 1.000 \\
$\quad$ Female & $24(34.3 \%)$ & $34(35.1 \%)$ & \\
Mean BMI (Kg/m $\left.{ }^{2}\right)^{a}$ & $22.3 \pm 2.3$ & $22.7 \pm 4.8$ & 0.495 \\
Preoperative comorbidity & & & 0.800 \\
$\quad$ None & $61(87.1 \%)$ & $87(89.7 \%)$ & \\
$\quad$ Hypertension & $6(8.6 \%)$ & $7(7.2 \%)$ & \\
$\quad$ Diabetes & $3(4.3 \%)$ & $3(3.1 \%)$ & \\
ASA & & & 0.794 \\
I-II & $64(91.3 \%)$ & $87(89.7 \%)$ & \\
III & $6(8.7 \%)$ & $10(10.3 \%)$ & \\
ESD preoperatively & & & 0.523 \\
Yes & $3(4.3 \%)$ & $7(7.2 \%)$ & \\
No & $67(95.7 \%)$ & $90(92.8 \%)$ & \\
\hline
\end{tabular}

$B M I$ body mass index, ASA American Society of Anesthesiologists, ESD endoscopic submucosal dissection

aalues are shown as mean \pm standard deviation

similar between LAPPG and LADG groups (Table 1). The perioperative outcomes are summarized in Table 2. The mean operation time and blood loss were comparable between two groups. For oncologic safety, there is no significant difference about the mean maximum tumor diameter, proximal margin, distal margin and no. of examined lymph between LAPPG and LADG group. The postoperative pathological examination revealed that all cases fulfilled pT1N0M0 criterion. Moreover, no difference was observed in median postoperative hospitalization, postoperative first flatus, postoperative gastric tube decompression, postoperative fluid diet start and Clavien Dindo Grade II or high complication. Nevertheless, hospitalization expenses were significantly less in LAPPG compared to LADG $(P=0.004)$.

\section{Postoperative nutrition, gallbladder contraction and gastric emptying}

The median follow up period was 24 months. As shown in Table 3, the levels of serum total protein, albumin, hemoglobin and BMI were significantly improved after LAPPG at 12 months compared to that of LADG. Table 4 summarized the functions of gallbladder contraction and gastric emptying in all patients. Gallstones developed in 5 LAPPG patients (7.1\%) and 5 LADG patients (5.2\%) without significant difference $(p=0.744)$. However, the mean gallbladder emptying rate was significantly higher in LAPPG group $(34.04 \pm 15.3$ vs $27.32 \pm 15.9)$ while the mean time to half gastric emptying $(110.11 \pm 44.5$ vs $92.51 \pm 54.6)$ and percentage of retention in the stomach 
Table 2 Perioperative outcomes and pathologic results about LAPPG and LADG

\begin{tabular}{|c|c|c|c|}
\hline Variables & $\operatorname{LAPPG}(n=70)$ & $\operatorname{LADG}(n=97)$ & $P$ value \\
\hline Mean operation time $(\min )^{b}$ & $220.5 \pm 17.2$ & $223.8 \pm 28.1$ & 0.216 \\
\hline Mean blood loss $(\mathrm{ml})^{\mathrm{b}}$ & $46.9 \pm 49.6$ & $48.5 \pm 51.1$ & 0.830 \\
\hline Mean maximum tumor diameter $(\mathrm{cm})^{\mathrm{b}}$ & $1.8 \pm 0.7$ & $1.8 \pm 0.7$ & 0.934 \\
\hline Mean proximal margin $(\mathrm{cm})^{\mathrm{b}}$ & $2.9 \pm 0.8$ & $3.0 \pm 0.8$ & 0.233 \\
\hline Positive proximal margin rate & $0(0 \%)$ & $0(0 \%)$ & 1.000 \\
\hline Mean distal margin $(\mathrm{cm})^{\mathrm{b}}$ & $3.8 \pm 1.4$ & $3.6 \pm 1.7$ & 0.265 \\
\hline Positive distal margin rate & $0(0 \%)$ & $0(0 \%)$ & 1.000 \\
\hline Mean total no. of examined lymph nodes ${ }^{b}$ & $22.4 \pm 5.3$ & $23.2 \pm 5.5$ & 0.337 \\
\hline Median postoperative hospitalization (days, range) & $8(7-30)$ & $8(7-27)$ & 0.199 \\
\hline Median postoperative first flatus (days, range) & $4(3-5)$ & $4(2-5)$ & 0.571 \\
\hline Median postoperative gastric tube decompression (days, range) & $4(2-20)$ & $3(2-19)$ & 0.656 \\
\hline Median postoperative fluid diet start (days, range) & $5(4-21)$ & $5(4-19)$ & 0.346 \\
\hline Postoperative complication ${ }^{a} \geq \|$ (no. of patients) & $11(15.7 \%)$ & $13(13.4 \%)$ & 0.824 \\
\hline Gastric stasis & $4(5.7 \%)$ & $2(2.1 \%)$ & \\
\hline Anastomotic leak & $1(1.4 \%)$ & $3(3.1 \%)$ & \\
\hline Abdominal absces & $1(1.4 \%)$ & $2(2.1 \%)$ & \\
\hline Respiratory complication & $5(7.1 \%)$ & $6(6.2 \%)$ & \\
\hline Differentiation & & & 0.215 \\
\hline Well or moderate differentiation & $47(67.1 \%)$ & $68(76.4 \%)$ & \\
\hline Poorly differentiation or Signet ring cell & $23(32.9 \%)$ & $21(23.6 \%)$ & \\
\hline pT category & & & 1.000 \\
\hline T1a & $33(47.1 \%)$ & $45(46.4 \%)$ & \\
\hline $\mathrm{T} 1 \mathrm{~b}$ & 37 (52.9\%) & $52(53.6 \%)$ & \\
\hline Hospitalization expenses (Ten thousands) ${ }^{b}$ & $4.6 \pm 0.5$ & $5.3 \pm 0.4$ & 0.004 \\
\hline
\end{tabular}

${ }^{a}$ Acording to the Clavien-Dindo classification

${ }^{b}$ Values are shown as mean \pm standard deviation

at $120 \min (46.27 \pm 20.5$ vs $40.27 \pm 21.9)$ were significantly more than those of LADG group $(p<0.05)$.

\section{Discussion}

PPG has been proved to be a safe operation for early gastric cancer patients with excellent short and long-term prognosis [16, 17]. LAPPG, a less invasive operation compared to PPG, not only had several advantages in early postoperative outcomes, such as reducing intraoperative blood loss, postoperative pain, hospital stay and accelerate bowel function recovery and fluid oral intake [18], but also could ameliorate early dumping syndromes, body weight loss and duodenogastric reflux although those patients might more frequently experience delayed gastric emptying, abdominal fullness and gastro-esophageal reflux disorder than LADG in short term $[8,19,20]$.

Since 2011, our surgical team started to apply laparoscopic approach to treat early gastric cancer, including LADG with D2 lymphadenectomy. After accumulating enough clinical experience, we started to apply LAPPG for cT1N0M0 gastric cancer patients whose tumor located in the middle portion of the stomach. In this study, we would like to share some experiences

Table 3 Nutritional status and BMI between LAPPG and LADG in one year follow-up

\begin{tabular}{llll}
\hline (Preoperative-postoperative)/preoperative & LAPPG $(n=70)$ & LADG $(n=97)$ & $P$ value \\
\hline Serum total protein level(\%) & $11.30 \pm 18.0$ & $5.9 \pm 13.0$ & 0.046 \\
Serum albumin level (\%) & $17.20 \pm 25.6$ & $10.06 \pm 15.4$ & 0.048 \\
Hemoglobin(\%) & $-1.09 \pm 4.0$ & $-2.73 \pm 3.8$ & 0.014 \\
BMI(\%) & $-2.53 \pm 3.1$ & $-3.64 \pm 3.4$ & 0.048 \\
\hline
\end{tabular}

Values are shown as mean $\pm S D, S D$ standard deviation, $B M I$ body mass index 
Table 4 Gallstone formation, gallbladder emptying and gastric emptying between LAPPG and LADG in one year follow-up

\begin{tabular}{llll}
\hline Variables & LAPPG $(n=70)$ & LADG $(n=97)$ & $P$ value \\
\hline Gallstone(n) & $5(7.1 \%)$ & $8(8.2 \%)$ & 1.000 \\
Gallbladder emptying rates(\%) & $34.04 \pm 15.3$ & $27.32 \pm 15.9$ & 0.012 \\
Time to half gastric emptying (min) & $110.11 \pm 44.5$ & $92.51 \pm 54.6$ & 0.032 \\
Retention at 120 min of stomach(\%) & $46.27 \pm 20.5$ & $40.27 \pm 21.9$ & 0.042 \\
\hline
\end{tabular}

Values are shown as mean \pm SD, SD standard deviation

regarding comparisons of short-term clinical outcomes as well as one year follow up surveillance of LAPPG and LADG in our institution. Furthermore, this is the first evaluation worldwide using objective methods such as gallbladder contraction and gastric emptying.

As shown in Table 1, the clinical characteristics of patients in LAPPG and LADG were similar. As far as perioperative outcomes and pathologic results are concerned, the mean operation time for LAPPG was $220.5 \pm 17.2 \mathrm{~min}$ and introperative blood loss was $46.9 \pm$ $49.6 \mathrm{ml}$, which were similar to other studies [18, 21, 22] and were not significantly different from those of LADG. Our results also showed that the postoperative recovery such as median hospitalization, postoperative fluid diet start, postoperative complications rate were not significantly different between LAPPG and LADG groups. Gastric stasis or delay emptying is a typical complication and might be the greatest pitfalls of PPG. In our study, 4 patients $(5.7 \%)$ in LAPPG group experienced gastric stasis while the number of LADG group is $2(2.1 \%)$ but there is no significant difference $(p=0.239)$. Also, our gastric stasis rate of LAPPG was comparable to the reports that ranged from 5.2 to $10.3 \%[8,21]$. The reasons for gastric stasis remain unclear. The possible mechanism was pylorus edema or nerve dysfunction induced by mechanical and chemical injury such as thermal insult from ultrasonic energy device $[18,23]$. The four gastric stasis patients in our study were all cured by conservative management such as gastric tube decompression, parenteral alimentation and fasting. Bae et al. [24] suggested that the standardization of the surgical procedure such as preserving blood flow and the hepatic branch of the vagus nerve could reduce the severity of gastric stasis. Zhu et al. [23] reported introperative manual dilation of pylorus help to prevent pyloric stenosis. However, those above skills were not tested in a randomized trial and thus a randomized controlled trial was needed to determine these outcomes.

In order to minimize interviewers' bias and obtain steady reproducibility, objective data including laboratory findings and BMI, instead of questionnaires from patients were collected in the outpatient clinic one year follow-up. As a result, the values of nutritional status such as serum total protein, albumin, hemoglobin and BMI in LAPPG group were elevated with significant

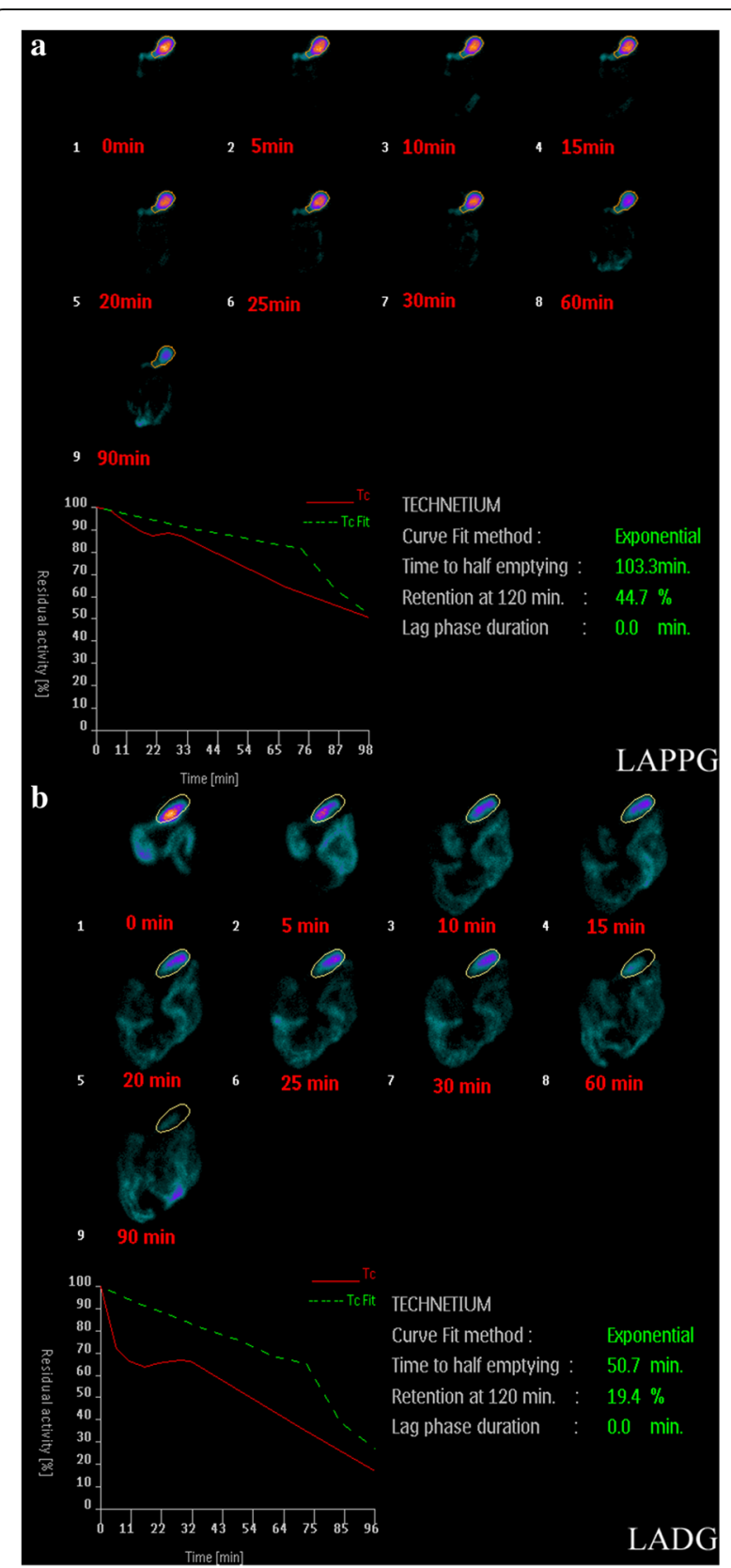

Fig. 1 Typical gastric emptying examination for LAPPG (a) and LADG (b) patients at one year follow-up surveillance respectively. a the time to half gastric emptying is $103.3 \mathrm{~min}$ and the retention at $120 \mathrm{~min}$ of stomach is $44.7 \%$. $\mathbf{b}$ the time to half gastric emptying is $50.7 \mathrm{~min}$ and the retention at $120 \mathrm{~min}$ of stomach is $19.4 \%$ 
difference as compared to those of LADG group. We speculated that the larger size functional gastric reservoir, preservation of pyloric cuff as well as hepatic and pyloric branches of vagus nerve, the retained gastric acid secretion and a longer gastric emptying for ferric iron and nutrition absorption after food mixed with gastric acid acted the beneficial factors in improving nutritional status and maintain BMI $[8,21,25,26]$.

The gastric emptying examination in one year follow-up further demonstrated the significantly longer time to half gastric emptying and more food retention at 120 min in LAPPG group than those in LADG group (Table 4 and Fig. 1).

Gallstone is one of common complications after gastrectomy [27]. The incidence of gallstone in LAPPG group was $7.1 \%$ and the gallbladder emptying rate was $34.04 \pm 15.3 \%$ in one year follow-up, both of which outdoes LADG group $(8.2 \%$ and $27.32 \pm 15.9 \%$, respectively). The pathophysiology of gallstone information after gastrectomy was regarded as vagal nerve resection, nonphysiological reconstruction of the gastrointestinal tract and decreased secretion of cholecystokinin [28, 29]. LAPPG could preserve hepatic and pyloric branches of the vagus nerve to keep pyloroduodenal myoneural continuity and maintain Oddi sphincter contraction and gallbladder emptying [30]. Findings from Imada et al. [25] and ours both proved that gallbladder functions in pylorus-preserving gastrectomy patients were much better than distal gastrectomy patients.

The most important limitation of our study was associated with its non-randomized, retrospective design. Nonetheless, this was the first report for comparing LAPPG to LADG in Chinese early gastric cancer patients and based on these retrospective experiences and data, our prospective randomized controlled trials (ClinicalTrials.gov Identifier: NCT02936193) are approved and starting to recruiting eligible patients which will present more detailed and persuasive studies. Secondly, the period of follow up was a little short considering the usual long-term survival analysis. However, the primary endpoint of our study was to evaluate clinical outcomes of surgery and nutrition objectively other than oncological safety which had already been tested and proved [3]. Finally, our study did not use questionnaires to evaluate QOL such as PGSAS-45 which was established by the Japanese Postgastrectomy Syndrome Working Party to measure postgastrectomy syndromes. That is because most of these subjective feelings from our small sample size study showed large dispersion degree.

\section{Conclusion}

In conclusion, our study demonstrated objective clinical outcomes of LAPPG for pT1N0M0 gastric in Chinese patients. By strict inclusion criteria, LAPPG could be a recommended surgical procedure for early gastric cancer located in the middle portion of the stomach. Ongoing clinical trials in our institution (ClinicalTrials.gov Identifier: NCT02936193) and Korean (ClinicalTrials.gov Identifier:NCT No.02595086) are expected to claim the indications and superiority of LAPPG.

\section{Abbreviations}

ASA: American Society of Anesthesiologists; ChiCTR: Chinese Clinical Trial Register; LADG: Laparoscopy-assisted distal gastrectomy; LAPPG: Laparoscopicassisted pylorus-preserving gastrectomy; PPG: Early gastric cancer (EGC); Pyloruspreserving gastrectomy

\section{Acknowledgements}

We kindly thank the editor and reviewers for careful review and valuable comments, which have led to a significant improvement of the manuscript.

\section{Funding}

Sponsored by Shanghai Sailing Program (Grant No. 17YF1415700); Shanghai Municipal Commission of Health and Family Planning (Grant No. 2017BR043); Hospital Development Center (Grant No. 16CR2022A). The funding bodies had no role in the design of the study and collection, analysis, and interpretation of data and in the writing of the manuscript.

\section{Availability of data and materials}

The datasets used and/or analysed during the current study are available from the corresponding author on reasonable request.

\section{Authors' contributions}

XX, CCZ have made substantial contributions to conception and design, acquisition of data, analysis and interpretation of data; FRY, JX have been involved in drafting the manuscript and revising it critically for important intellectual content; GZ, HC have made substantial contributions to acquisition of data, analysis and interpretation of data. All authors have read and approved the manuscript.

\section{Ethics approval and consent to participate}

Ethics approval was obtained by the Ethics Committee of Ren Ji hospital and written informed consent for participation in the study was obtained from participants. Research was in compliance with the Declaration of Helsinki (https://www.wma.net/policies-post/wma-declaration-of-helsinki-ethicalprinciples-for-medical-research-involving-human-subjects/).

\section{Consent for publication}

Not applicable

\section{Competing interests}

The authors declare that they have no competing interests.

\section{Publisher's Note}

Springer Nature remains neutral with regard to jurisdictional claims in published maps and institutional affiliations.

Received: 15 December 2018 Accepted: 8 May 2019

Published online: 22 May 2019

\section{References}

1. Torre LA, Bray F, Siegel RL, et al. Global cancer statistics, 2012. CA Cancer J Clin. 2015;65:87-108.

2. Ahn HS, Lee HJ, Yoo MW, et al. Changes in clinicopathological features and survival after gastrectomy for gastric cancer over a 20-year period. Br J Surg. 2011;98:255-60.

3. Oh SY, Lee HJ, Yang HK. Pylorus-preserving gastrectomy for gastric Cancer. J Gastric Cancer. 2016;16:63-71.

4. Kim YW, Baik YH, Yun YH, et al. Improved quality of life outcomes after laparoscopy-assisted distal gastrectomy for early gastric cancer: results of a prospective randomized clinical trial. Ann Surg. 2008;248:721-7.

5. Adachi Y, Suematsu T, Shiraishi N, et al. Quality of life after laparoscopyassisted Billroth I gastrectomy. Ann Surg. 1999;229:49-54. 
6. Kim HH, Hyung WJ, Cho GS, et al. Morbidity and mortality of laparoscopic gastrectomy versus open gastrectomy for gastric cancer: an interim report-a phase III multicenter, prospective, randomized trial (KLASS trial). Ann Surg. 2010;251:417-20.

7. Uyama I, Okabe H, Kojima K, et al. Gastroenterological surgery: stomach. Asian J Endosc Surg. 2015;8:227-38.

8. Suh YS, Han DS, Kong SH, et al. Laparoscopy-assisted pylorus-preserving gastrectomy is better than laparoscopy-assisted distal gastrectomy for middle-third early gastric cancer. Ann Surg. 2014;259:485-93.

9. Maki T, Shiratori T, Hatafuku T, et al. Pylorus-preserving gastrectomy as an improved operation for gastric ulcer. Surgery. 1967;61:838-45.

10. Japanese Gastric Cancer Association. Japanese gastric cancer treatment guidelines 2014 (ver. 4). Gastric Cancer. 2017;20(1):1-19.

11. Xia X, Zhang Z, Xu J, et al. Comparison of postoperative lymphocytes and interleukins between laparoscopy-assisted and open radical gastrectomy for early gastric cancer. J Int Med Res. 2018. https://doi.org/10.1177/0300060518800909.

12. Japanese Gastric Cancer Association. Japanese classification of gastric carcinoma: 3rd English edition. Gastric Cancer. 2011;14(2):101-112.

13. Sobin LH. Frequently asked questions regarding the application of the TNM classification. TNM/prognostic factors project (International Union against Cancer [UICC]). Cancer. 1999;85:1405-6.

14. Dindo D, Demartines N, Clavien PA. Classification of surgical complications: a new proposal with evaluation in a cohort of 6336 patients and results of a survey. Ann Surg. 2004;240:205-13.

15. Dodds WJ, Groh WJ, Darweesh RM, et al. Sonographic measurement of gallbladder volume. AJR Am J Roentgenol. 1985;145:1009-11.

16. Morita S, Katai H, Saka M, et al. Outcome of pylorus-preserving gastrectomy for early gastric cancer. Br J Surg. 2008;95:1131-5.

17. Hiki N, Sano T, Fukunaga T, et al. Survival benefit of pylorus-preserving gastrectomy in early gastric cancer. J Am Coll Surg. 2009;209:297-301.

18. Jiang $X$, Hiki N, Nunobe $S$, et al. Postoperative outcomes and complications after laparoscopy-assisted pylorus-preserving gastrectomy for early gastric cancer. Ann Surg. 2011;253:928-33.

19. Hosoda K, Yamashita K, Sakuramoto S, et al. Postoperative quality of life after laparoscopy-assisted pylorus-preserving gastrectomy compared with laparoscopy-assisted distal gastrectomy: a cross-sectional postal questionnaire survey. Am J Surg. 2017:213:763-70.

20. Nunobe S, Sasako M, Saka M, et al. Symptom evaluation of long-term postoperative outcomes after pylorus-preserving gastrectomy for early gastric cancer. Gastric Cancer. 2007;10:167-72.

21. Tsujiura M, Hiki N, Ohashi M, et al. Excellent long-term prognosis and favorable postoperative nutritional status after laparoscopic pyloruspreserving gastrectomy. Ann Surg Oncol. 2017;24:2233-40.

22. Jiang $X$, Hiki N, Nunobe $\mathrm{S}$, et al. Long-term outcome and survival with laparoscopy-assisted pylorus-preserving gastrectomy for early gastric cancer. Surg Endosc. 2011;25:1182-6.

23. Zhu CC, Kim TH, Berlth F, et al. Clinical outcomes of intraoperative manual dilatation of pylorus in pylorus-preserving gastrectomy: a retrospective analysis. Gastric Cancer. 2018.

24. Bae JS, Kim SH, Shin Cl, et al. Efficacy of gastric balloon dilatation and/or retrievable stent insertion for pyloric spasms after pylorus-preserving gastrectomy: retrospective analysis. PLoS One. 2015;10:e0144470.

25. Imada T, Rino Y, Takahashi M, et al. Postoperative functional evaluation of pylorus-preserving gastrectomy for early gastric cancer compared with conventional distal gastrectomy. Surgery. 1998;123:165-70.

26. Kodama M, Koyama K, Chida T, et al. Early postoperative evaluation of pylorus-preserving gastrectomy for gastric cancer. World J Surg. 1995;19: 456-60 discussion 461

27. Hauters $\mathrm{P}$, de neve de Roden A, Pourbaix A, et al. Cholelithiasis: a serious complication after total gastrectomy. Br J Surg. 1988;75:899-900.

28. Kobayashi T, Hisanaga M, Kanehiro $\mathrm{H}$, et al. Analysis of risk factors for the development of gallstones after gastrectomy. Br J Surg. 2005;92:1399-403.

29. Inoue K, Fuchigami A, Hosotani R, et al. Release of cholecystokinin and gallbladder contraction before and after gastrectomy. Ann Surg. 1987;205: $27-32$.

30. Nabae T, Takahata S, Konomi H, et al. Effect of prepyloric gastric transection and anastomosis on sphincter of Oddi cyclic motility in conscious dogs. J Gastroenterol. 2001:36:530-7.

\section{Ready to submit your research? Choose BMC and benefit from:}

- fast, convenient online submission

- thorough peer review by experienced researchers in your field

- rapid publication on acceptance

- support for research data, including large and complex data types

- gold Open Access which fosters wider collaboration and increased citations

- maximum visibility for your research: over $100 \mathrm{M}$ website views per year

At BMC, research is always in progress.

Learn more biomedcentral.com/submissions 